ISSN electrónico: 2445-1355

DOI: https://doi.org/10.14201/fj2021622737

\title{
ATENCIÓN FARMACÉUTICA EN PROBIÓTICOS Y ESTIMULACIÓN INMUNITARIA EN UNA OFICINA DE FARMACIA DE SALAMANCA DURANTE LOS MESES DE SEPTIEMBRE A ENERO DE 2020
}

\section{Pharmaceutical Care in Probiotics and Immune Stimulation in a Pharmacy Located in Salamanca from September to January 2020}

Isabel RODRÍGUEZ; Crisanto MARTÍN*

Farmacia comunitaria Elvira Martín. Calle María Auxiliadora, 74. 37004 Salamanca. Correo-e: crisantomartin@redfarma.org*

RESUMEN: El sistema inmune y la microbiota constituyen la primera línea de defensa del organismo contra patógenos, por lo que su buen funcionamiento es esencial para nuestra salud.

La microbiota es el conjunto de los numerosos microorganismos que residen en nuestro organismo, y cuando esta se ve alterada se pueden desarrollar diversas patologías. Por ello, los probióticos, prebióticos y simbióticos tienen un papel muy importante como tratamiento preventivo o como coadyuvante ayudando a reequilibrar nuestra microbiota, además de poseer un destacable efecto inmunomodulador.

Para recomendar o consumir un producto probiótico de forma adecuada debemos tener en cuenta la cepa y la dosis para cada patología a tratar, ya que, de lo contrario, la eficacia podría no ser la esperada.

En este estudio se tratará de comprobar el conocimiento y el uso de los probióticos en la población de una farmacia comunitaria, verificar su 
eficacia y detectar posibles efectos adversos, además de fomentar la educación sanitaria por parte del farmacéutico.

Palabras clave: probiótico; microbiota; sistema inmune; atención farmacéutica.

ABSTRACT: The immune system and the microbiota are the body's first line of defence against pathogens, so their proper functioning is essential for our health.

The microbiota is the set of numerous microorganisms that reside in our body, and when it is altered, various pathologies can develop. Therefore, probiotics, prebiotics and synbiotics play a very important role as a preventive or adjuvant treatment, helping to rebalance our microbiota, as well as having a significant immunomodulatory effect.

When recommending or consuming a probiotic product, we must take into account the strain and dose for each patology to be treated, otherwise the efficacy may not be as expected.

The aim of this study is to test the knowledge and use of probiotics in the population of a community pharmacy, to verify their efficacy and to detect possible adverse effects, as well as to promote health education by the pharmacist.

Keywords: probiotic; microbiota; immune system; pharmaceutical care.

\section{INTRODUCCIÓN}

\subsection{La microbiota}

Nuestro organismo está formado por numerosas bacterias que crean un auténtico ecosistema, denominado microbiota. La microbiota autóctona se define como el conjunto de comunidades microbianas que colonizan establemente en diferentes cavidades del organismo. Cada una, con una variedad y densidad microbiana específica, siendo única y propia en cada individuo. Se localizan en las fosas nasales, la piel, el aparato genitourinario, el aparato respiratorio y el aparato digestivo, siendo el intestino grueso donde la concentración y cantidad bacteriana es mayor (Suárez, 2013). 


\subsection{Funciones de la microbiota}

Tiene un papel importante en la protección contra agentes infecciosos (antagonismo microbiano) y en el desarrollo y modulación del sistema inmune, así como la diferenciación del epitelio de la mucosa intestinal (funciones tróficas). También lleva a cabo funciones metabólicas, como la producción de ácidos grasos de cadena corta (acético, propiónico y butírico), producción de vitamina $\mathrm{K}$ y ayuda a mejorar la absorción de calcio y hierro².

Normalmente existe una simbiosis mutualista, pero puede hacerse parasitaria debido a alteraciones de nuestra microbiota habitual. Esto se denomina disbiosis.

\subsection{Factores que influyen en la microbiota}

La colonización bacteriana del intestino comienza tras el nacimiento, aunque existen estudios que corroboran que hay una exposición intraútero con los microorganismos de la madre, alcanzando un patrón adulto a los 2 años.

La microbiota residente va a depender de factores genéticos, especialmente de la microbiota materna, el tipo de parto (natural o cesárea) y la alimentación (leche materna o fórmulas).

Con el crecimiento del individuo será relevante la dieta, el ambiente, toma de antibióticos u otros fármacos, infecciones y/o enfermedades que se desarrollen. En una edad anciana, la microbiota estará relacionada con el estado fisiológico del individuo.

Todos estos factores van a influir tanto cualitativa como cuantitativamente en la composición de nuestra microbiota.

\subsection{Probióticos, prebióticos y simbióticos}

Los probióticos se definen según la OMS como "microorganismos vivos que, al ser administrados en cantidades adecuadas, confieren un beneficio a la salud en el huésped”. Para considerarse probióticos deben mostrar (Guillot, 2018):

- Propiedades no patógenas.

- Capacidad de supervivencia a través del tracto digestivo.

- Adherencia al epitelio intestinal.

- Colonización en el tracto intestinal.

- Producción de sustancias antimicrobianas.

- Estabilidad en forma farmacéutica o en alimentos. 
Los prebióticos son compuestos no digeribles, presentes en la dieta, que estimulan el crecimiento o la actividad de los microorganismos autóctonos aportando un efecto beneficioso en el consumidor. Los simbióticos se definen como mezcla de probióticos y prebióticos. Solo se denomina simbiótico si su efecto beneficioso es superior a la suma de sus integrantes.

\subsection{Probióticos y sistema inmune}

Los probióticos son buenos aliados en el mantenimiento de la composición, el metabolismo y la inmunidad de la microbiota. Pueden modular el sistema inmune no solo a nivel intestinal, también a nivel sistémico.

Los mecanismos de acción que llevan a cabo son:

- Inhibición competitiva evitando la colonización de patógenos. Además, crean un entorno hostil para su supervivencia, produciendo ácido láctico $\mathrm{O}_{2} \mathrm{O}_{2}$, que acidifican el medio, o secretando antimicrobianos como bacteriocinas.

- Refuerzan la función de la barrera intestinal que impide la entrada de patógenos a la lámina propia. Además, producen AGCC, principal fuente de energía de colonocitos, que colaboran con la integridad de la barrera.

- Interaccionan con células del sistema inmune como macrófagos y células dendríticas, que secretan citocinas, que regulan fundamentalmente la función de células T reguladoras para inducir inmunomodulación sistémica disminuyendo la susceptibilidad a la inflamación (Singh et al., 2018).

\subsection{Indicaciones}

El mecanismo de acción de los probióticos difiere mucho no solamente entre especies, sino entre ciertas cepas. Por lo que la eficacia de su uso dependerá de la cepa, dosis, vía de administración, modo de conservación o el estado de salud del consumidor.

Se emplean para prevención de patologías o como coadyuvantes de otros tratamientos.

Los efectos adversos tras el consumo de probióticos son escasos, aunque se requiere especial atención en aquellos pacientes inmunodeprimidos (Rodríguez, 2015).

Ediciones Universidad de Salamanca / @@@ FarmaJournal, vol. 6, núm. 2 (2021), pp. 27-37 
ISABEL RODRÍGUEZ; CRISANTO MARTÍN

ATENCIÓN FARMACÉUTICA EN PROBIÓTICOS Y ESTIMULACIÓN INMUNITARIA EN UNA OFICINA DE FARMACIA DE SALAMANCA DURANTE LOS MESES DE SEPTIEMBRE A ENERO DE 2020

TABLA 1. Resumen de las diversas indicaciones y los beneficios con mayor evidencia de los probióticos

(Barro et al., 2018; Guarner et al., 2017)

\begin{tabular}{|c|c|c|}
\hline Indicación & Dosis recomendada & Cepa \\
\hline $\begin{array}{l}\text { Diarrea aguda en la } \\
\text { infancia }\end{array}$ & $\begin{array}{l}250-750 \mathrm{mg} / \text { día } \\
10^{8} \mathrm{UFC} / \text { día } \\
10^{10} \mathrm{UFC} / \text { día }\end{array}$ & $\begin{array}{l}\text { Saccharomyces boulardii } \\
\text { CNCM I-745 } \\
\text { Lactobacillus reuteri DMS } \\
17938 \\
\text { L. rhamnosus GG }\end{array}$ \\
\hline $\begin{array}{l}\text { Diarrea aguda en } \\
\text { adultos }\end{array}$ & $\begin{array}{l}10^{9} \text { UFC, dos veces/día } \\
5 \times 10^{9} \mathrm{UFC} \text {, dos veces/día }\end{array}$ & $\begin{array}{l}\text { Lactobacillus paracasei } \mathrm{B} 21060 \\
\text { o L. rhamnosus GG } \\
\text { S. boulardii CNCM I-745 }\end{array}$ \\
\hline $\begin{array}{l}\text { Diarrea asociada a } \\
\text { antibióticos }\end{array}$ & $\begin{array}{l}10^{8} \text { UFC, dos veces/día } \\
10^{10} \mathrm{UFC} \text {, dos veces/día }\end{array}$ & $\begin{array}{l}\text { Lactobacillus reuteri DMS } \\
17938 \\
\text { L. rhamnosus GG }\end{array}$ \\
\hline $\begin{array}{l}\text { Prevención diarrea del } \\
\text { viajero }\end{array}$ & $\begin{array}{l}10^{9} \mathrm{UFC} / \text { día } \\
2-6 \times 10^{9} \mathrm{UFC} \text {, una o dos } \\
\text { veces/día }\end{array}$ & $\begin{array}{l}\text { L. acidophilus, L. bulgaricus, } L \text {. } \\
\text { bifidum y S. thermophilus } \\
\text { S. boulardi CNCM I-745 }\end{array}$ \\
\hline Cólicos del lactante & $\begin{array}{l}10^{8} \mathrm{UFC} / \text { día, } 5 \text { gotas/día } \\
10^{9} \mathrm{UFC} / \text { día, } 5 \text { gotas/día }\end{array}$ & $\begin{array}{l}\text { L. reuteri DMS } 17938 \\
\text { Bifidobacterium longum y } \\
\text { Pediococcus pentosaceus }\end{array}$ \\
\hline $\begin{array}{l}\text { SII (Síndrome } \\
\text { Intestino Irritable) }\end{array}$ & $\begin{array}{l}5 \times 10^{7} \mathrm{UFC} / \text { día } \\
10^{8} \mathrm{UFC} / \text { día }\end{array}$ & $\begin{array}{l}\text { L. plantarum, } 299 v \\
\text { Bifidobacterium longum } \\
\text { infantis } 35624\end{array}$ \\
\hline $\begin{array}{l}\text { Enfermedad } \\
\text { inflamatoria intestinal: } \\
\text { colitis ulcerosa }\end{array}$ & $\begin{array}{l}0.25-5 \times 10^{10} \text { UFC, una o dos } \\
\text { veces/día } \\
5 \times 10^{9} \text { UFC, dos veces al día }\end{array}$ & $\begin{array}{l}\text { E. coli } \\
\text { S. boulardii CNCM I-745 }\end{array}$ \\
\hline Helicobacter pylori & $\begin{array}{l}6 \times 10^{9} \text { UFC, dos veces al día } \\
1 \times 10^{8} \text { UFC, tres veces al día }\end{array}$ & $\begin{array}{l}\text { L. rhamnosus GG } \\
\text { L. reuteri DMS } 17938\end{array}$ \\
\hline $\begin{array}{l}\text { Estreñimiento } \\
\text { funcional }\end{array}$ & $1 \times 10^{8}$ UFC, dos veces/día & L. reuteri DMS 17938 \\
\hline $\begin{array}{l}\text { Mala absorción de la } \\
\text { lactosa }\end{array}$ & $\begin{array}{l}\text { Mínimo } 10^{8} \text { UFC de cada } \\
\text { cepa por gramo de producto }\end{array}$ & $\begin{array}{l}\text { Streptococcus thermophilus, } B . \\
\text { longum } 15708, \text { L. reuteri DMS } \\
17938 \text { (uso microorganismos } \\
\text { del yogur) }\end{array}$ \\
\hline Vulvovaginitis & $10^{8}$ UFC vía vaginal & L. rhamnosus + L. gasseri \\
\hline
\end{tabular}

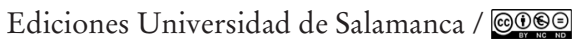


ISABEL RODRÍGUEZ; CRISANTO MARTÍN

ATENCIÓN FARMACÉUTICA EN PROBIÓTICOS Y ESTIMULACIÓN INMUNITARIA EN UNA OFICINA

DE FARMACIA DE SALAMANCA DURANTE LOS MESES DE SEPTIEMBRE A ENERO DE 2020

\begin{tabular}{l|l|l}
\multicolumn{1}{c|}{ Indicación } & \multicolumn{1}{c|}{ Dosis recomendada } & \multicolumn{1}{c}{ Cepa } \\
\hline Mastitis & $3 \times 10^{9} \mathrm{UFC}$ & L. fermentum CECT 5716 \\
\hline $\begin{array}{l}\text { ITU (infecciones } \\
\text { tracto urinario) }\end{array}$ & $10^{9} \mathrm{UFC/día}$ & $\begin{array}{l}\text { L. plantarum CECT 8675 y } \\
\text { CECT 8677 }\end{array}$ \\
\hline $\begin{array}{l}\text { Dermatitis atópica } \\
\text { Salud bucodental } \\
\begin{array}{l}\text { gingivitis, halitosis, } \\
\text { caries) }\end{array}\end{array}$ & $5 \times 10^{8} \mathrm{UFC}, 1-2$ veces/día & $\begin{array}{l}\text { L. rhamnosus LR05 } \\
\text { B. lactis BS01 }\end{array}$ \\
\hline
\end{tabular}

\subsection{Papel de farmacéutico}

Para elegir un preparado tendremos en cuenta las características y necesidades del paciente: forma farmacéutica (gotas, polvos, comprimidos, cápsulas, óvulos) y/o alergias e intolerancias (gluten, lactosa...).

Se debe evitar su ingesta simultánea con alimentos o bebidas muy calientes, y con un margen de al menos 2 horas, para la toma de antibióticos.

Generalmente, se debe conservar por debajo de los $22-25^{\circ} \mathrm{C}$ y protegidos de la luz, y, en algunos casos, refrigerados entre los $2-8^{\circ} \mathrm{C}$.

\section{2. Овjetivo}

El objetivo principal de este trabajo es evaluar el uso y conocimiento de la población sobre los probióticos, prebióticos y simbióticos, que se llevará a cabo en una farmacia comunitaria.

Otros objetivos a conseguir serán:

- Educación sanitaria a la población acerca de los probióticos, fomentando el papel del farmacéutico en el desarrollo de la atención farmacéutica.

- Detección de posibles grupos de riesgo.

- Verificar la eficacia de la toma de los probióticos para los problemas de salud requeridos.

\section{Metodología}

Para llevar a cabo este trabajo han sido entrevistados 28 pacientes en una farmacia comunitaria, durante el periodo de septiembre a enero de 2021. 
La entrevista constaba de dos partes: una primera encuesta acerca del conocimiento que el paciente tenía sobre los probióticos y su situación de salud. Además, se le hacía entrega de un tríptico informativo en relación con el tema abordado.

La segunda encuesta se realizó para comprobar si el tratamiento indicado había sido efectivo y si el paciente resultaba satisfecho. Para ello, en los casos en los que el paciente no era cliente habitual de la oficina de farmacia, ha sido necesario realizarla a través de llamada telefónica.

Todos los datos han sido recopilados con ayuda de la herramienta Microsoft Excel.

Al mismo tiempo, ha sido necesaria una búsqueda bibliográfica para el contenido teórico.

\section{Resultados y discusión}

La mayoría de los encuestados han resultado mujeres, el $82 \%$ de la muestra. La edad que predominaba era de entre 25 a 65 años con un porcentaje de un $61 \%$.

Los pacientes solían acudir a la farmacia pidiendo un probiótico específico que le había recomendado el médico o porque ya lo habían tomado con anterioridad y conocían la pauta indicada. A pesar de ello, algunos afirmaron que desconocían todos los beneficios que abarcaba el uso de probioticos.

En ocasiones se solicitaba consejo del farmacéutico, sobre todo dudas acerca de la duración y pautas a la hora de tomar el probiótico.

Por otra parte, a veces fue el farmacéutico quién aconsejaba el uso de probióticos al paciente, como en tratamientos con antibióticos, indicando que ayudaría a restablecer el equilibrio de la microbiota y en caso de posible diarrea u otros efectos adversos, remitiendo los síntomas.

Es cierto que algunos declinaron nuestra recomendación, sobre todo personas mayores, ya que añadir otro medicamento a su medicación habitual no les satisfacía. O en los casos en los que era otra persona la que iba a recoger la medicación del paciente.

Según los resultados, el $79 \%$ de los pacientes entrevistados ya contaban con cierto conocimiento del uso de probióticos y un 64 \% los había tomado con anterioridad (Figura 1). Dentro de los pacientes que los habían tomado, la mayoría indicaron que su uso fue beneficioso para su salud y no tuvieron ningún efecto adverso. 


\section{Conocimiento sobre probióticos}
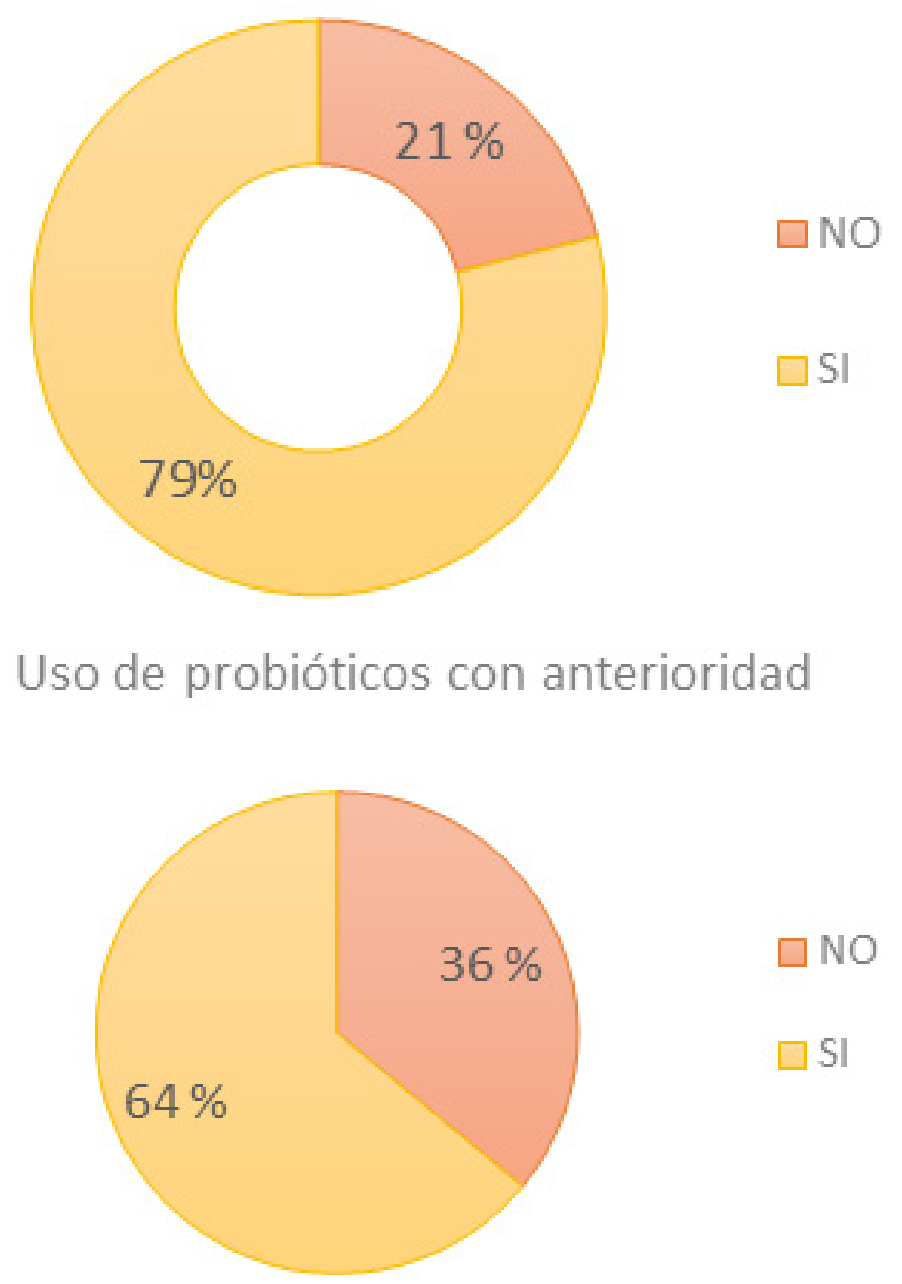

Figura 1. Porcentaje sobre el conocimiento previo del uso de probióticos y porcentaje de pacientes que ya habían recibido un tratamiento con probióticos.

Ediciones Universidad de Salamanca / @@ $\quad$ FarmaJournal, vol. 6, núm. 2 (2021), pp. 27-37 


\section{Motivo de consulta}

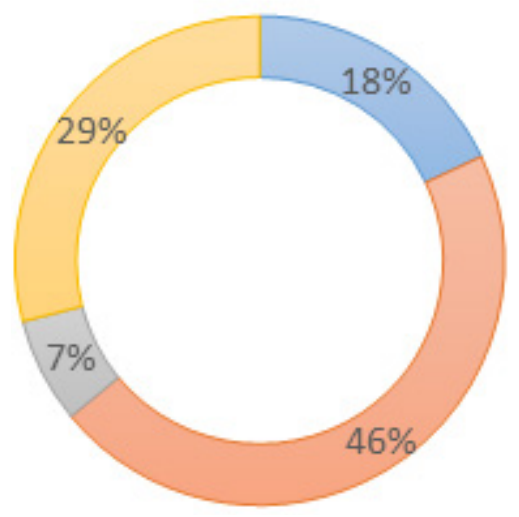

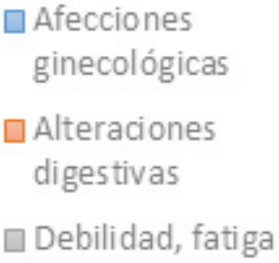

$\square$ Uso de antibióticos

Figura 2. Motivo del uso de los probióticos en los pacientes entrevistados.

El motivo de consulta más recurrente ha sido debido a alteraciones digestivas, siendo un $77 \%$ sucesos de estreñimiento o diarrea. Otras patologías que se trataron fueron casos de cólico del lactante, gastritis y Helicobacter pylori.

Respecto a afecciones genitourinarias, destacó la prevención de episodios de infecciones de orina recurrentes en un $60 \%$. En este caso, se optaba por un producto que incluyese arándano rojo americano. En un $40 \%$ se trataron casos de candidiasis.

El uso de antibióticos se utilizó en casos de otitis, infección de orina y otras infecciones bacterianas. Los probióticos se utilizaron como tratamiento preventivo para combatir los efectos secundarios de los antibióticos, como es la diarrea, en la mayoría de los casos.

También hubo un $7 \%$ que utilizaron probióticos con el fin de aumentar las defensas, que, además, no conocían con anterioridad este tipo de terapia.

Otros datos recopilados fueron las patologías previas y el consumo de otros medicamentos, que podrían ser útiles para relacionar en caso de posible efecto adverso.

Además, se les preguntó acerca de sus hábitos, pues malos hábitos de salud están muy relacionados con una microbiota desequilibrada y, por lo tanto, un sistema inmune poco fortalecido.

Dentro de los pacientes que afirmaron llevar a cabo unos hábitos nocivos, destacó el estrés y el tabaco con un $27 \%$ y un $26 \%$ respectivamente, siguiendo con 
un $20 \%$ que indicaron ausencia de ejercicio físico; y, en último lugar, un $14 \%$ y un $13 \%$ con una dieta poco saludable y falta de descanso, respectivamente. Cabría investigar si estos datos tienen influencia por la situación actual de pandemia por el virus SARS-Covid-2.

Por ello, al entregar el tríptico informativo, se hizo hincapié en cuáles y cómo unos hábitos más saludables ayudarían a sentirnos mejor y mejorar nuestra salud.

Consideramos, aprovechando la campaña de vacunación de la gripe, averiguar qué pacientes estaban vacunados. Los resultados fueron que el $61 \%$ no estaban vacunados frente al $39 \%$ que sí estaban vacunados.

Hay estudios que afirman que el uso de probióticos podría ser una manera segura, eficiente y fácil para mejorar la respuesta inmune provocada por la vacunación antigripal en grupos (Barro et al., 2018; Vitetta et al., 2017). En este caso, es difícil poder afirmar dicha correlación, más cuando los pacientes mayores de 65 años a los que se les recomendó vacunarse son escasos en este estudio. De cualquier forma, no ha habido grandes diferencias en cuanto a los beneficios del uso de los probióticos entre personas vacunadas y las que no.

Hubo un caso de una mujer en periodo de lactancia, la cual no reportó efecto adverso. Se ha visto que el uso de probióticos durante el tercer trimestre de embarazo o lactancia puede ayudar al desarrollo de la microbiota intestinal del bebé, disminuyendo incidencia de alergias; incluso, si la madre tiene antecedentes de dermatitis atópica, reduce el riesgo en el niño (Barro et al., 2018).

Los resultados de la segunda encuesta fueron satisfactorios. La mayoría siguió el tratamiento según la pauta establecida y obtuvo mejoras en su salud, sin identificar ningún efecto adverso. Solo hubo dos casos a destacar: una paciente con tratamiento de $H$. pylori afirmó que notó mejoría, pero, al suprimir la toma del probiótico, los síntomas negativos reaparecieron. Otro caso en el que la paciente no obtuvo mejora, pero tampoco efectos adversos.

De todos modos, la mayoría afirmaban que volverían a utilizarlos en caso de necesidad y que les sirvió de ayuda la información obtenida.

\section{Conclusiones}

1. Según los resultados se podría afirmar que la población sí tiene conocimiento sobre el uso probióticos.

2. El uso de probióticos es efectivo y seguro en las diversas patologías tratadas.

3. No existe una relación causal aparente entre el uso de probióticos y la concomitancia de la vacuna de la gripe.

4. Considero que es un tipo de tratamiento con mucha visión de futuro y que necesita aún más investigación. 
5. Hubiese sido interesante constatar si más a largo plazo el uso de los probióticos, acompañado de unos hábitos más saludables, dieran como resultado una mejora de salud del individuo, de tal forma que enfermase menos e incluso se sintiera con mejor energía y ánimo.

6. Los pacientes reconocieron útil la información obtenida confirmando la labor imprescindible del farmacéutico. De hecho, una parte de la muestra optó por la terapia con probióticos para estimular su sistema inmune.

\section{Bibliografía}

Barro AM, Sáez AC, Mateos Lardiés AM, Sampedro AR, Sánchez AM, Suárez Fernandez JE et al. Guía de actuación y documento de consenso sobre el manejo de preparados con probióticos y/o prebióticos en la farmacia comunitaria SEFAC y SEPyP [Internet] SEFAC y SEPyP; 2018. Disponible en: https://www.sefac.org/sites/default/ files/2018-07/GUIA_PROBIOTICOS\%20WEB.pdf

Guarner F. Papel de la microbiota intestinal en la salud y en la enfermedad. Nutr Hosp 2007; 22: supl. 2.

Guarner F, Sanders ME, Eliakim R, Fedorak R, Gangl A, Garisch J et al. Guías mundiales de la Organización Mundial de la Gastroenterología. Probióticos y prebióticos. World Gastroenterology Organisation (WGO); 2017. Disponible en: https://www. worldgastroenterology.org/UserFiles/file/guidelines/probiotics-and-prebioticsspanish-2017.pdf

Guillot CC. Probióticos, puesta al día. Rev Cubana Pediatr. 2018; 90:2.

Ottman N, Smidt H, de Vos WM, Belzer C. The function of our microbiota: who is out there and what do they do? 2012; 2:104.

Rodríguez J M, Probióticos: del laboratorio al consumidor. Nutr Hosp. 2015; 31 supl. $1: 33-47$.

Singh Y, Ahmad J, Musarrat J, Ehtesham NZ, Hasnain SE. Emerging importance of holobionts in evolution and in probiotics. 2013; 5: 12.

Suárez JE. Microbiota autóctona, probióticos y prebióticos. Nutr Hosp. 2013; 28: supl. 1.

Vitetta L, Saltzman ET, Thomsen M, Nikov T, Hall S. Adjuvant probiotics and the intestinal microbiome: enhancing vaccines and immunotherapy outcomes. 2017; 5(4):50. 
